\title{
Single Pulse Heating of Nanoparticle Array for Biological Applications
}

Chen Xie, ${ }^{\dagger}$ Peiyuan Kang, ${ }^{\dagger}$ Johan Cazals, ${ }^{\dagger}$ Omar Morales Castelán, ${ }^{\dagger}$ Jaona Randrianalisoa, ${ }^{*}$ Zhenpeng Qin**,\$,/l

${ }^{\dagger}$ Department of Mechanical Engineering, University of Texas at Dallas, 800 West Campbell Road, Richardson, Texas 75080, United States.

Institut de Thermique, Mécanique, Matériaux (ITheMM EA 7548), University of Reims Champagne-Ardenne, Reims, Cedex 2 51687, France.

${ }^{\S}$ Department of Bioengineering, Department of Bioengineering, Center for Advanced Pain Studies, University of Texas at Dallas, 800 West Campbell Road, Richardson, Texas 75080, United States.

"Department of Surgery, University of Texas at Southwestern Medical Center, 5323 Harry Hines Boulevard, Dallas, Texas 75390, United States.

\section{AUTHOR INFORMATION:}

Corresponding Author: Zhenpeng Qin*

Department of Mechanical Engineering, University of Texas at Dallas, 800 West Campbell Road EW31, Richardson, Texas 75080, United States.

E-mail: Zhenpeng.Qin@UTDallas.edu 
ORICD: 0000-0003-3406-3045

\section{KEYWORDS:}

nanoparticle heating, nanoscale-confined heating, macroscale-collective heating, protein denaturation, TRPV1 channel activation 


\begin{abstract}
:
With the ability to convert external excitation into heat, nanomaterials play an essential role in many biomedical applications. Two modes of nanoparticle (NP) array heating, nanoscaleconfined heating $(\mathrm{NCH})$ and macroscale-collective heating $(\mathrm{MCH})$, have been found and extensively studied. Despite this, the resulting biological response at protein level remains elusive. In this study, we developed a computational model to systematically investigate the single-pulsed heating of NP array and corresponding protein denaturation/activation. We found that $\mathrm{NCH}$ may lead to targeted protein denaturation, however, nanoparticle heating does not lead to nanoscale selective TRPV1 channel activation. The excitation duration and NP concentration are primary factors that determine a window for targeted protein denaturation, and together with heating power, we defined quantified boundaries for targeted protein denaturation. Our results boost our understandings in the $\mathrm{NCH}$ and $\mathrm{MCH}$ under realistic physical constraints and provide a robust guidance to customize biomedical platforms with desired NP heating.
\end{abstract}




\section{Introduction}

Nanomaterials can be designed to efficiently absorb optical or magnetic energy and convert that energy to heat. ${ }^{[1-3]}$ This heat-generating property has found applications in cancer treatment, ${ }^{[4-8]}$ drug delivery, ${ }^{[9,10]}$ contrast imaging, ${ }^{[11-14]}$ vision restoration, ${ }^{[15]}$, pain $^{[16]}$ and neuromodulation (Figure S1). ${ }^{[17,18]}$ While these advances have pushed the boundaries of biomedicine, there has been a fair amount of debate over the past few years in understanding the effect of heating in biological systems, such as in the area of magnetogenetics on whether magnetothermal heating of ferritin nanoparticles can actually generate enough heat to actuate thermally-sensitive ion channels. ${ }^{[19-23]}$ Scaling analysis suggests that the heat generated by ferritin is 9-10 order of magnitude less than that threshold to activate TRPV1 ion channels ${ }^{[24]}$ and recent reports suggest a biochemical pathway involving reactive oxygen species (ROS) that activates both TRPV1 and TRPV4 ion channels. ${ }^{[23,25]}$ This highlights the importance of understanding nanoscale heating and its impact on biological systems, especially proteins.

Heating generated by nanomaterials broadly falls into one of two regimes: nanoscale-confined heating $(\mathrm{NCH})$ and macroscale-collective heating $(\mathrm{MCH}) . \mathrm{NCH}$ is characterized by local temperature increases at the nanoscale which remain confined near the surface of the nanoparticle, as in the case of controlling protein activity by molecular hyperthermia, ${ }^{[15,16,26]}$ or membrane permeability by opto-poration. ${ }^{[16,26-28]}$ In contrast, $\mathrm{MCH}$ is characterized by a widespread temperature increase throughout the medium, such as whole cells or tissues. $\mathrm{MCH}$ can, for example, be used to injure cancer cells, ${ }^{[5]}$ excite thermally-sensitive neurons expressing transient receptor potential (TRP) channels, ${ }^{[15]}$ and regulate cellular function. ${ }^{[29]}$ Keblinski et al. highlighted the role of the duration of energy excitation in controlling the transition between $\mathrm{NCH}$ and $\mathrm{MCH},{ }^{[30]}$ demonstrating that short energy pulses could lead to $\mathrm{NCH}$ while continuous 
excitation could cause MCH. Similarly, Baffou et al. systemically investigated the boundary and transition between temperature confinement (or $\mathrm{NCH}$ ) and delocalization (or $\mathrm{MCH}$ ) under continuous excitation and repeated femtosecond pulsed excitations, respectively. ${ }^{[31,}$ 32] Furthermore, methods for generating arbitrary microscale temperature profiles have been explored using NPs and spatial light modulation. ${ }^{[33]}$ While these efforts advanced our understanding of the spatiotemporal evolution of nanoparticle heating, there is an important gap in understanding how single pulsed NP heating affects biological systems through protein denaturation (by hyperthermia ${ }^{[5]}$ or molecular hyperthermia ${ }^{[16]}$ ) or activation (for example thermally sensitive ion channels TRPV1). ${ }^{[15]}$

In this report, we investigated the heating of NP arrays under single pulse excitation and the resulting protein responses, including protein thermal denaturation and activation of thermallysensitive ion channels. Our analysis suggests that the excitation duration and NP area density determine a window for heterogeneous protein denaturation, where targeted denaturation is possible. Together with heating power, we defined boundaries for targeted protein denaturation inside this heterogeneous denaturation window. For activation of thermally sensitive ion channel TRPV1, we determined that the nanoparticle array heating leads to widespread of channel activation instead of localized activation, because heat dissipates and leads to $\mathrm{MCH}$ within milliseconds, a timescale required to activate the ion channel based on our current understanding. ${ }^{[34]}$ This work provides guidance for designing innovative approaches that utilize $\mathrm{NCH}$ and $\mathrm{MCH}$ for modulating molecular or tissue-specific activities under realistic physical constraints.

\section{Results and Discussions}




\subsection{NP array heating characteristics and corresponding protein denaturation}

We first constructed and validated the modeling framework for NP array heating and protein thermal denaturation (Figure $1 \mathrm{~A}$ ). For our model systems, we used $10 \mu \mathrm{m} \times 10 \mu \mathrm{m} 2 \mathrm{D}$ square arrays composed of $30 \mathrm{~nm}$ gold nanoparticles (AuNP) immersed in water, which were designed to mimic NPs that are targeted to a cell membrane. The NP array heating was acquired by a superposition method (Equation S1). Our results suggest that different NP materials with the same heat generation will have a similar heating profile in water (Figure S2 A), which is the main target for analyzing heating in biological tissues. Therefore, we used heating power per NP ( $g$ ) that is independent of NP absorption properties to represent the heat generation of NPs by different methods, such as laser or magnetic field (Figure S1). This heating model was used throughout the remaining of the paper.

Heating can trigger a series of biological responses, including protein denaturation in the case of cancer thermal therapy, ${ }^{[6,35]}$ and activation of temperature-sensitive TRPV1 channels that modulate neuron activity. ${ }^{[15,36]}$ Recently, Kang et al. showed the possibility of nanoscale selective protein denaturation with AuNP targeting and nanosecond pulsed laser excitation. ${ }^{[16]}$ To connect NP heating to the protein denaturation, we first analyzed protein denaturation using $\alpha$ chymotrypsin since its denaturation properties have been measured in both $\mathrm{NCH}$ and $\mathrm{MCH} .{ }^{[28,37]}$ Protein thermal denaturation can be modeled as a temperature-sensitive chemical reaction (Equation S3, Figure $1 \mathrm{~A}$ ), and the normalized concentration of the denatured protein $(D)$ is determined by the reaction rate and reaction duration (Equation S4). Since temperature profile and protein denaturation are all time dependent, we consider the end of excitation duration as the representative time point $(t)$ for temperature change $(\Delta T)$ analysis, and the time after the laser pulse $(10 \times$ excitation duration $)$ as the representative time point for protein denaturation analysis. 
Similarly, we further consider the NP-water interface $\left(\mathrm{P}_{1}\right)$ and the mid-point between NPs $\left(\mathrm{P}_{2}\right)$ as two representative locations for $\Delta T$ and denaturation analysis (Figure $\mathrm{S} 2 \mathrm{~B}$ ). We will use $\mathrm{P}_{1}$ and $\mathrm{P}_{2}$ to indicate these locations, along with $\Delta T_{1}, \Delta T_{2}$ and $D_{1}, D_{2}$ to indicate temperature increase $(\Delta T)$ and normalized protein denaturation $(D)$ at $\mathrm{P}_{1}$ and $\mathrm{P}_{2}$ respectively. These representative time points and locations are used throughout the paper. With this framework, we examined the characteristics of nanoscale-confined heating $(\mathrm{NCH})$ and macroscale-collective heating $(\mathrm{MCH})$ and connected them to corresponding protein denaturation. The thermal activation of TRPV1 was analyzed using a similar framework and presented in later sections. Figure $1 \mathrm{~B}$ shows that $\mathrm{NCH}$ was observed under short excitation duration (10 ns) and low NP area density $\left(9 \mu \mathrm{m}^{-2}\right)$, where heating is confined to the nanoparticle surface (Figure S3 A-B). As a result, targeted protein denaturation is established where the protein denaturation was confined to the vicinity of the NPs (Figure1 B, Figure S3 A-B). By increasing the heating duration to $1 \mu$ s and the NP area density to $100 \mu^{-2}$ (Figure $1 \mathrm{C}$ ), the heating fully dissipates and leads to $\mathrm{MCH}$ where the temperature increased uniformly. In this case, widespread protein denaturation was observed. Together, these two cases demonstrate the major hallmarks of $\mathrm{NCH}$ and $\mathrm{MCH}$ and the resulting targeted versus widespread protein denaturation. 
A
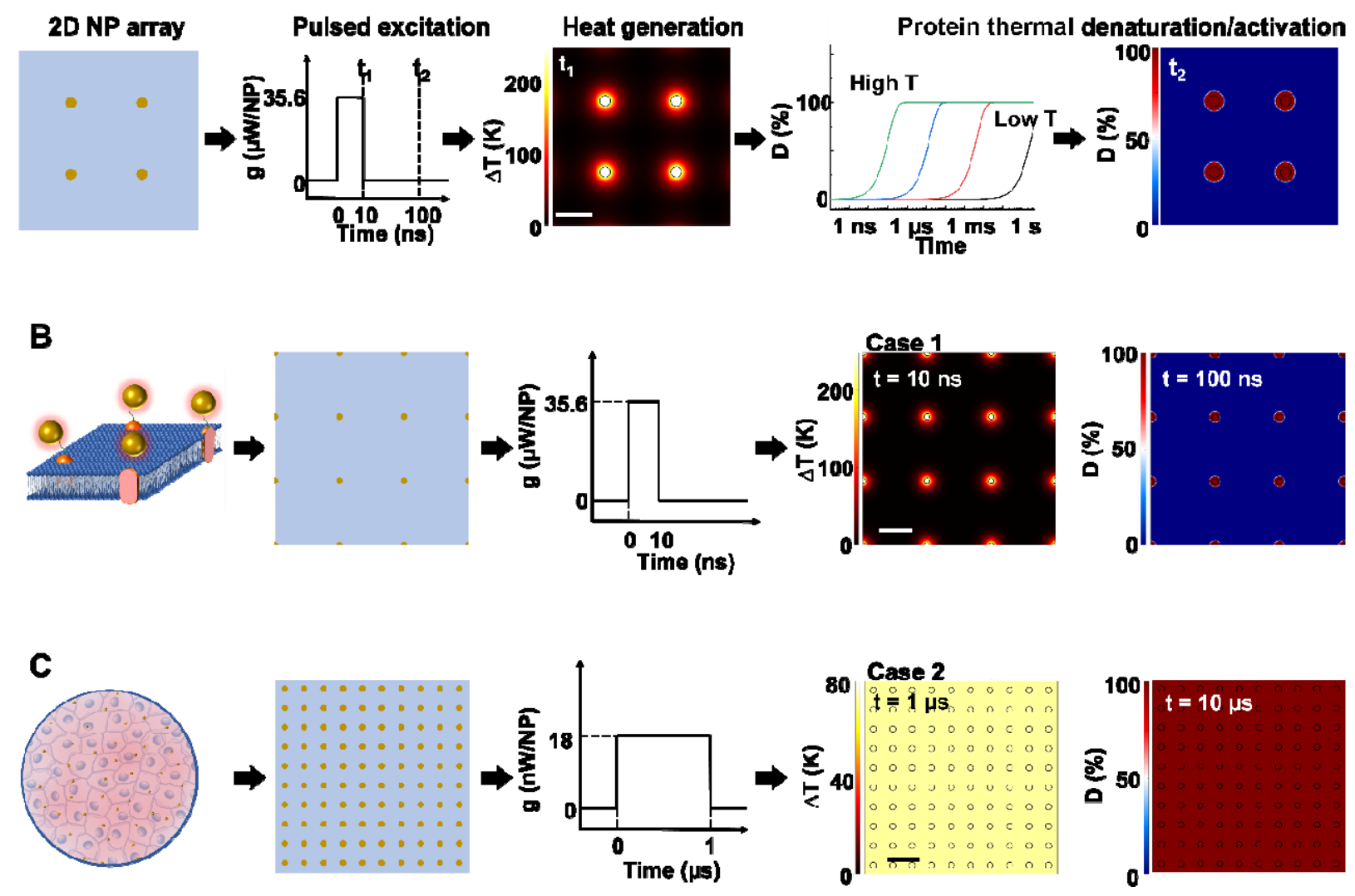

Figure 1. Analysis framework for nanoparticle heating-induced protein denaturation and characteristics of protein denaturation under nanoscale-confined heating (NCH) and macroscale-collective heating (MCH). (A) Schematic of the analysis framework for nanoparticle (NP) array heating and the resulting protein denaturation $(D)$ or activation. External excitation of the NP array (each NP as a nano heater) heats up the NP and the surrounding media. The temperature increase drives a chemical reaction (protein denaturation or activation), which can be described by first-order kinetic model (the Arrhenius model). Integration of the reaction rate over time leads to an overall protein denaturation or activation. (B-C) Nanoscale confined heating $(\mathrm{NCH})$ and macroscale collective heating $(\mathrm{MCH})$, and the corresponding temperature increase and protein denaturation. Conditions: domain size $10 \mu \mathrm{m} \times 10 \mu \mathrm{m}, \mathrm{NP}$ diameter $\left(d_{N P}\right)=$ 
$30 \mathrm{~nm}$, heating power per NP $(g)$ is (B) $35.6 \mu \mathrm{W}$ and (C) $18 \mathrm{nW}, \mathrm{NP}$ area density is (B) $9 \mu \mathrm{m}^{-2}$ and (C) $100 \mu \mathrm{m}^{-2}$, and excitation duration is (B) $10 \mathrm{~ns}$ and (C) $1 \mu \mathrm{s}$.

\subsection{Key factors that affect the NP array heating modes and protein denaturation}

Next, we identified the key factors that impact the transition from $\mathrm{NCH}$ to $\mathrm{MCH}$ and from targeted to widespread protein denaturation. We first investigated the role of excitation duration by comparing the thermal responses and corresponding protein denaturation of an NP array (area density of $9 \mu \mathrm{m}^{-2}$ ) at three distinctive excitation durations (Figure $2 \mathrm{~A}$; case 3: $10 \mathrm{~ns}$, case 4: 100 ns, and case 5: $1 \mu \mathrm{s}$; all under same heating power $(g=35.6 \mu \mathrm{W})$; summary of all cases in Table S3). Here we adopt $\Delta T_{2} / \Delta T_{1}$ and $D_{2} / D_{1}$ to characterize the temperature change and denaturation profile. High $\Delta T_{2} / \Delta T_{1}$ and $D_{2} / D_{1}$ indicate uniform $\Delta T$ and denaturation profiles, while low $\Delta T_{2} / \Delta T_{1}$ and $D_{2} / D_{1}$ indicate heterogeneous profiles. Figure 2 B-D illustrate the $\Delta T$ profiles and protein denaturation for these cases. For case $3(10 \mathrm{~ns})$, heating was confined and $\Delta T_{2}$ remained constant (Figure $2 \mathrm{~B} \& \mathrm{D}$ ), which resulted in low $\Delta T_{2} / \Delta T_{1}$ (Figure $2 \mathrm{E}$ ); while for cases 4 and 5 (100 ns and $1 \mu \mathrm{s}), \Delta T_{2}$ increases significantly, leading to an increase of $\Delta T_{2} / \Delta T_{1}$. Cases $3 \& 4$ (10 and 100 ns excitation) resulted in targeted denaturation with low $D_{\text {total }}(1.6 \%$ and $4.8 \%$ respectively , Equation S5) and $D_{2} / D_{1}(\sim 0 \%)$. In contrast, Case $5(1 \mu \mathrm{s})$ exhibited widespread protein denaturation $\left(D_{\text {total }}=99 \%, D_{2} / D_{1}=100 \%\right)$. In this scenario, we observed a sharp increase in $\Delta T_{2} / \Delta T_{1}, D_{2} / D_{1}$ and $D_{\text {total }}$ when the excitation duration increases (Case 5, Figure $2 \mathrm{E}$ ). Therefore, the excitation duration is considered as a key factor in both transitions (i.e., from $\mathrm{NCH}$ to $\mathrm{MCH}$ and from targeted to widespread denaturation).

When targeting and manipulating protein receptors on the cell surface, the NP area density depends on the receptor density. For example, metabotropic glutamate receptors (mGluRs) have 
an area density of $2 \sim 51 \mu \mathrm{m}^{-2},{ }^{[2,13]}$ requiring a similar NP area density on the cell surface for effective targeting. To evaluate the role of NP area density, we compared thermal responses and protein denaturation under three different NP arrays with distinct NP area densities (Figure 2 F; case $6,2.6 \mu \mathrm{m}^{-2}$; case $7,25 \mu \mathrm{m}^{-2}$; case $8,100 \mu \mathrm{m}^{-2}$; all under identical excitation condition: excitation duration $20 \mathrm{~ns}, g=35.6 \mu \mathrm{W}$ ). Figure $2 \mathrm{G}-\mathrm{H}$ shows that, at low and medium area densities $\left(2.6 \mu \mathrm{m}^{-2}\right.$ and $\left.25 \mu \mathrm{m}^{-2}\right), \mathrm{NCH}$ and targeted protein denaturation is observed. In contrast, at high area density $\left(100 \mu \mathrm{m}^{-2}\right)$, heating generated by adjacent NPs are overlapped, resulting in $\mathrm{MCH}\left(\Delta T_{2} / \Delta T_{1}=34.2 \%\right)$ and widespread denaturation $\left(D_{\text {total }}=100 \%, D_{2} / D_{1}=100 \%\right)$. Based on this analysis, we consider the NP area density as another key factor in both transitions.
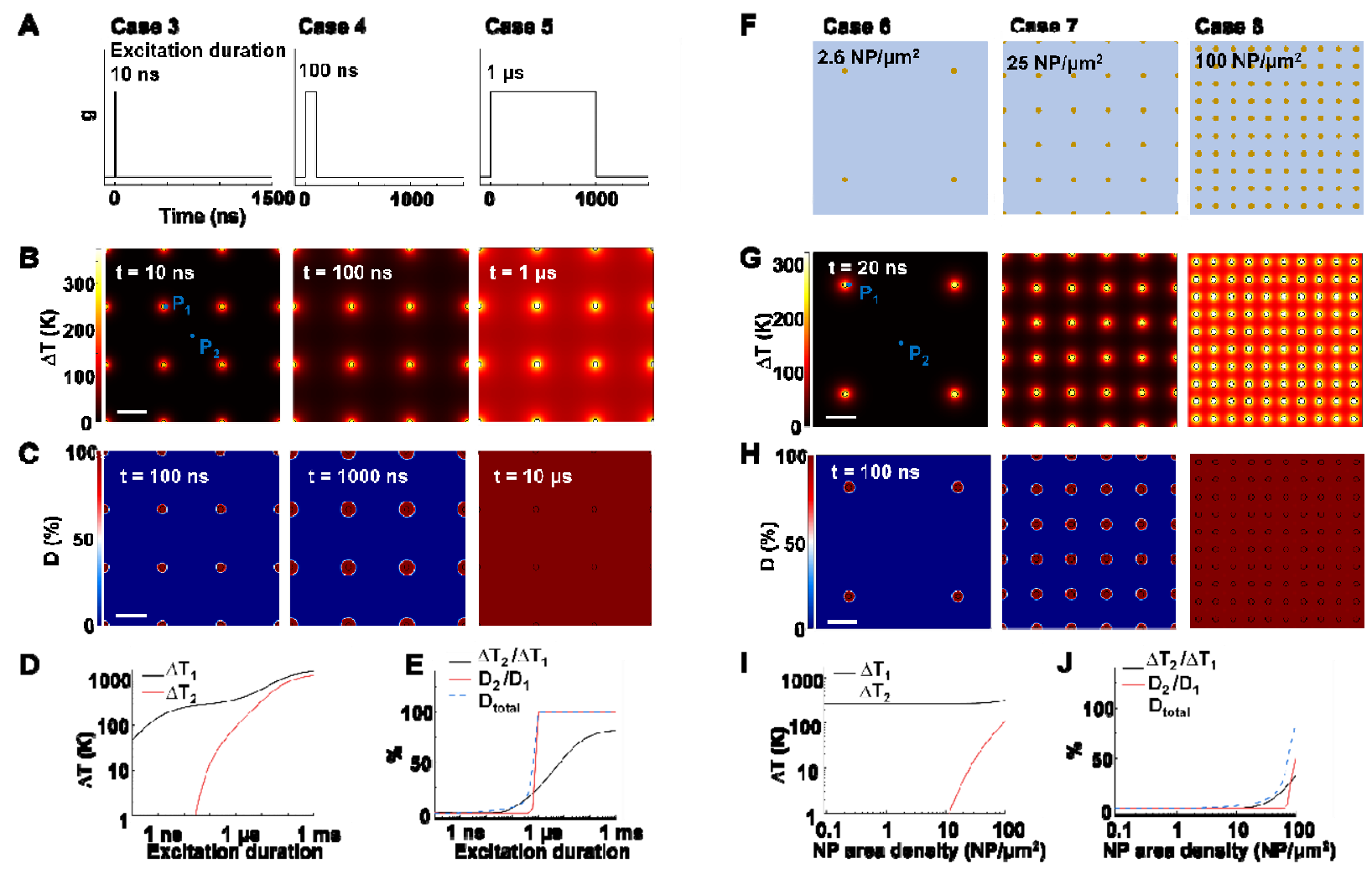

Figure 2. Effects of excitation duration and NP area density on the heating and protein

denaturation. (A) Schematic of excitation durations for case 3-5. (B) $\Delta T$ profile and (C) 
protein denaturation $(D)$ profile for cases $3-5$. For case $3-5, \mathrm{NP}$ area density is at $9 \mu \mathrm{m}^{-2} . \mathrm{P}_{1}$ represents the NP-water interface, $\mathrm{P}_{2}$ represents mid-point between NPs. (D) $\Delta T$ at $\mathrm{P}_{1}\left(\Delta T_{1}\right)$ and $\Delta T$ at $\mathrm{P}_{2}\left(\Delta T_{2}\right)$ as a function of excitation duration. (E) Ratio between $\Delta T_{2}$ and $\Delta T_{1}\left(\Delta T_{2} / \Delta T_{1}\right)$, ratio between protein denaturation at $\mathrm{P}_{2}$ and $\mathrm{P}_{1}\left(D_{2} / D_{1}\right), D_{\text {total }}$ in terms of excitation duration. $(\mathrm{F})$ Schematic of NP area density for cases 6-8 at $2.6 \mu \mathrm{m}^{-2}, 25 \mu \mathrm{m}^{-2}$, and $100 \mu \mathrm{m}^{-2}$, respectively. (G) $\Delta T$ profile and (H) $D$ profile for cases 6-8. For cases 6-8, excitation duration $20 \mathrm{~ns}$. (I) $\Delta T_{1}$ and $\Delta T_{2}$ in terms of NP area density. (J) $\Delta T_{2} / \Delta T_{1}, D_{2} / D_{1}$ and $D_{\text {total }}$ in terms of NP area density. For all cases, $g=35.6 \mu \mathrm{W}$ and scalebar represents $200 \mathrm{~nm}$.

The spatial distribution of NPs on the cell membrane is dependent on the distribution of target receptors, which can be randomly distributed. ${ }^{[13,38]}$ To evaluate the role of NP distribution, we examined thermal responses and protein denaturation for three different NP arrays with identical area density $\left(9 \mu \mathrm{m}^{-2}\right)$ distributed in square (case 9), hexagonal (case 10) and random (case 11) patterns (Figure S4 A). When subjected to a $20 \mathrm{~ns}$ excitation at $35.6 \mu \mathrm{W} / \mathrm{NP}$, all of the NP arrays exhibited $\mathrm{NCH}$ and targeted protein denaturation (Figure S4 B\&C). However, a close examination revealed that random distribution causes local temperature hot spots at the regions with NP aggregations (Figure S4 and Figure S5). This agrees with previous reports which showed that local temperature can be much higher around NP clusters. ${ }^{[39,40]}$ It worth pointing out that $D_{\text {total }}$ of all three cases are similar due to the few local hot spots, making the NP distribution less significant for protein denaturation. 
A

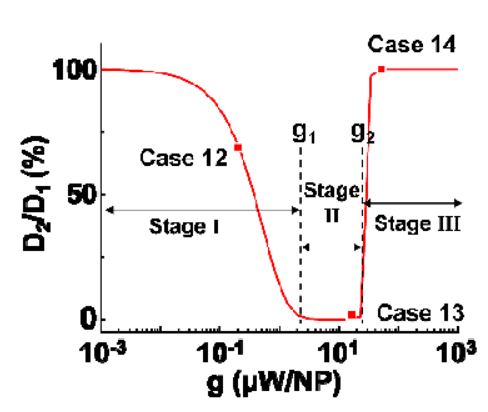

C

$\mathbf{E}$

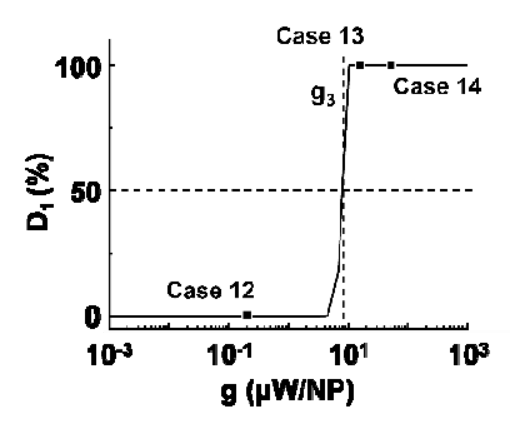

B

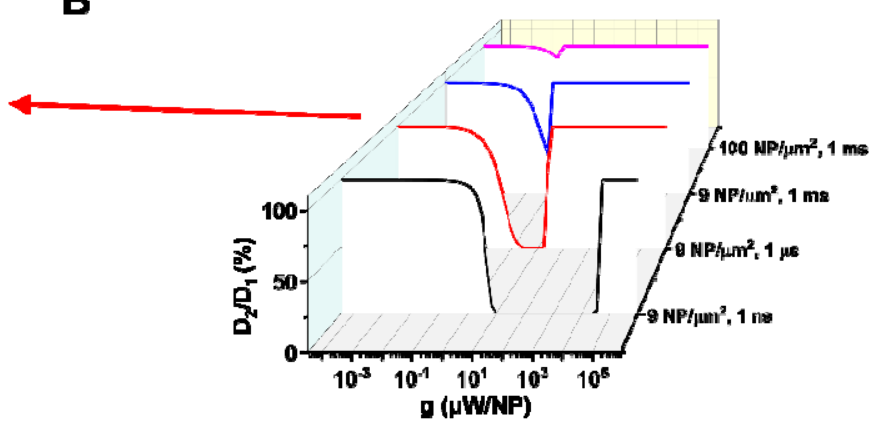

D

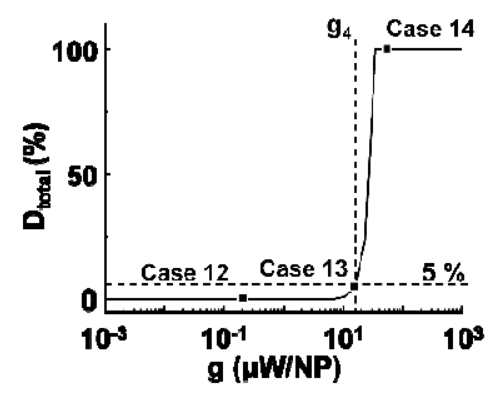

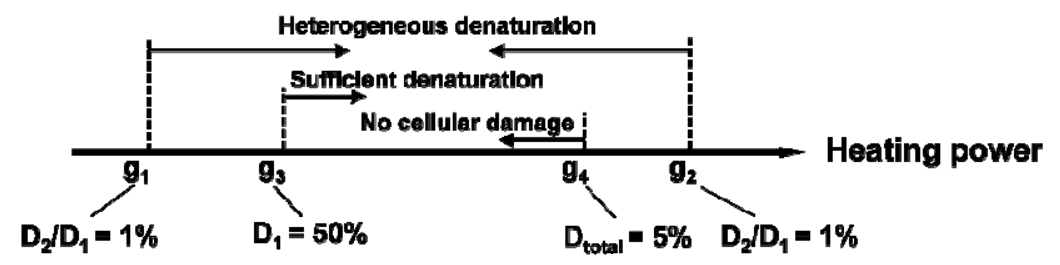

Figure 3. NP heating power determines a window for targeted denaturation. (A) $D_{2} / D_{1}$ in terms heating power $(g)$ with a $1 \mu$ s excitation duration and a $9 \mathrm{NP} / \mu \mathrm{m}^{2}$ area density. Three major stages for protein denaturation as $g$ increases: Stage $\square$, uniformly no denaturation zone with high $D_{2} / D_{1}(1 \%-100 \%)$; Stage $\square$, targeted denaturation window with low $D_{2} / D_{1}(<1 \%)$; Stage $\square$, widespread denaturation with high $D_{2} / D_{1}(1 \%-100 \%) . g_{1}$ and $g_{2}$ are critical heating powers that separate the three stages. (B) $D_{2} / D_{1}$ in terms of $g$ with different combinations of excitation durations and NP area densities. (C) $D_{l}$ in terms $g$ with a $1 \mu$ s excitation duration and a $9 \mu \mathrm{m}^{-2}$ area density. Here we define a critical heating power $g_{3}$ that corresponds $D_{l}=50 \%$. (D) $D_{\text {total }}$ in 
terms $g$ with a $1 \mu$ s excitation duration and a $9 \mu \mathrm{m}^{-2}$ area density. We define a critical heating power $g_{4}$ that corresponds $D_{\text {total }}=5 \%$. (E) Schematic for $g_{1}, g_{2}, g_{3}$, and $g_{4}$.

Next, we analyzed the effect of heating power $(g)$ on the protein denaturation. $\Delta T$ increased linearly with $g$ (Table $S$ 1-2), resulting in a similar $\Delta T$ profile with different magnitude. On the other hand, the corresponding denaturation increased nonlinearly as per the Arrhenius equation (Equation S3). Here we compared cases under different heating powers with identical excitation duration $(1 \mu \mathrm{s})$ and NP area density $\left(9 \mu \mathrm{m}^{-2}\right)$. We identified three stages (I-III) of protein denaturation based on how $D_{2} / D_{1}$ changes with the $g$. Figure 3 A and Figure S6 show that, in Stage I (homogeneous intact stage, case 12 as an example), there is no significant protein denaturation throughout the system due to insufficient $g$ (both $D_{2}$ and $D_{1}$ are small, leading to high $D_{2} / D_{1}$ ratio). In the Stage II, $g$ is sufficient to generate a localized hot area and significant denaturation around NPs (case 13 as an example), with no significant heating or protein denaturation at $\mathrm{P}_{2}\left(D_{2} \sim 0 \%\right)$. Therefore, a heterogeneous denaturation profile was observed with a small $D_{2} / D_{1}$ ratio $(<1 \%$ ). In the Stage III (case 14 as an example), $g$ is sufficient to heat up the whole system and denature all proteins regardless of position, leading to homogeneous denaturation. We thus define two critical heating powers, $g_{1}$ and $g_{2}$ at $D_{2} / D_{1}=1 \%$ as boundaries for heterogeneous denaturation (Figure $3 \mathrm{~A}$ ). It is worth noting that $g_{1}$ and $g_{2}$ are dependent on excitation duration and NP area density (Figure 3 B). Stage II vanishes at high NP area density and excitation duration (Figure $3 \mathrm{~B}, 100 \mu \mathrm{m}^{-2}, 1 \mathrm{~ms}$ ), with a direct transition from no denaturation to widespread denaturation. This agrees with previous sections, where long excitation duration and high NP area density lead to $\mathrm{MCH}$ (high $\Delta T_{2} / \Delta T_{1}$ ). As such, there is no window for heterogeneous or targeted denaturation. To quantify the threshold for sufficient denaturation at $\mathrm{P}_{1}$, we further define a heating power $g_{3}$ corresponding to $D_{1}=50 \%$ (Figure $3 \mathrm{C}$ ). 
Heating power that is much lower than $g_{3}$ does not produce sufficient denaturation around NPs. Furthermore, studies have suggested a large total protein denaturation can lead to cellular damage (e.g. $\left.D_{\text {total }}=5 \%\right)^{41}$. Therefore, we define critical heating power $g_{4}$ corresponding to $D_{\text {total }}=5 \%$ (Figure $3 \mathrm{D}$ ) as threshold for cellular damage. Figure 3 E summaries the definitions for $g_{1}, g_{2}, g_{3}$, and $g_{4}$. It is notable that relations between $g_{1}, g_{2}, g_{3}$, and $g_{4}$ may change when altering excitation duration and NP area density.

With the above analysis, the targeted denaturation should meet three criteria simultaneously:

(1) A heterogeneous denaturation $\left(g_{1}<\right.$ heating power $\left.<g_{2}\right)$;

(2) Sufficient denaturation at $\mathrm{P}_{1}$ (heating power $>g_{3}$ );

(3) Minimal $D_{\text {total }}$ to avoid cellular damage (heating power $<g_{4}$ ).

Bases on these criteria above, we analyzed a large combination of NP arrays and excitations by altering the excitation durations $(0.1 \mathrm{~ns}-1 \mathrm{~ms}), \mathrm{NP}$ area densities $\left(0.09-100 \mu \mathrm{m}^{-2}\right)$, and heating powers $\left(10^{-3}-10^{9} \mu \mathrm{W}\right)$. First, we generate a 3D map for the boundaries of heterogeneous denaturation. Figure S7 A shows critical surfaces corresponding to $g_{1}$ and $g_{2}$, where the intersection (dotted line) indicates absence of heterogeneous denaturation. Projecting this dotted line in a 2D plot (Figure $4 \mathrm{~A}$ ) shows the limit for the heterogeneous denaturation window, where targeted denaturation is possible. Next, we investigated boundaries for the second and third criterion located in this window. Figure S7 B\&C show critical surfaces of $g_{3}$ and $g_{4}$ respectively, where targeted denaturation should between surfaces of $g_{3}$ and $g_{4}$ to achieve sufficient denaturation while avoiding cellular damage. Lastly, we combined all the boundaries discussed above and generated a 3D map for targeted protein denaturation (Figure $4 \mathrm{~B}$ ). A lower limit (maximum of $g_{l}$ and $g_{3}$ ) and an upper limit (minimum of $g_{2}$ and $g_{4}$ ) can be set, within which there 
is targeted denaturation. For example, our results suggest that $\mathrm{NCH}$ is established for the case of $10 \mathrm{~ns}$ excitation duration at $9 \mu \mathrm{m}^{-2}$, and target denaturation can be achieved for a heating power within $14-81 \mu \mathrm{W}$. In contrast, for the case of $1 \mathrm{~ms}$ excitation duration at $100 \mu \mathrm{m}^{-2}$, targeted denaturation does not exist regardless of the heating power since $\mathrm{MCH}$ is established. It should be emphasized that these results are based on $\alpha$-chymotrypsin, while other proteins may have different thresholds. However, we anticipate a similar trend for other proteins when the Arrhenius model is applied. NCH can lead to novel applications in selectively manipulating protein activity by molecular hyperthermia, ${ }^{[16,26]}$ or membrane permeability, ${ }^{[27,28]}$ On the other hand, macroscale-collective heating results in uniform heating and widespread protein denaturation for applications such as cancer thermal therapy. ${ }^{[4,5]}$ Therefore, our analysis provides a framework and guideline to achieve targeted protein denaturation while avoiding widespread damage.
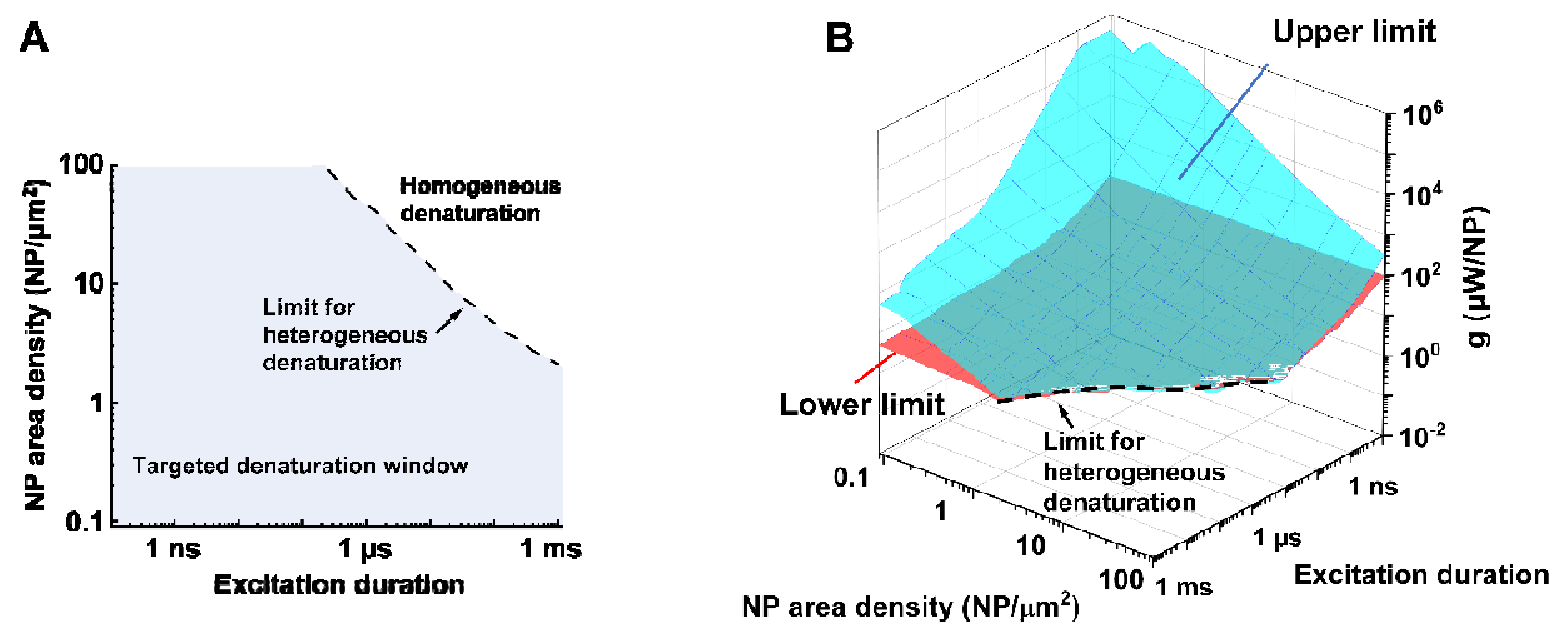

Figure 4. Limits and window for targeted protein denaturation (A) Projection of the limit for heterogeneous protein denaturation (dotted line), Region below the line (blue area) demonstrated the window for heterogeneous protein denaturation and possible targeted denaturation provided 
with propriate heating power (shown in B). (B) Lower limit (red surface) and upper limit (blue surface) in terms of excitation duration and NP area density. Region between red surface and blue surface indicates window for targeted protein denaturation.

\subsection{NCH is insufficient for TRPV1 activation}

Lastly, we investigated whether NP array heating could selectively activate TRPV1 channel. TPRV1 channel activation can be treated as a heat-activated chemical reaction described by a two-state model (Equation S6-S9). Previous studies have demonstrated that the TRPV1 channel can be activated within milliseconds at temperatures ranging from $40-53{ }^{\circ} \mathrm{C}$ (Figure $\left.5 \mathrm{~A}\right) .{ }^{[34,42]}$ Following the framework in Figure 5A, we further investigated TRPV1 activation by NP array heating within $40-53{ }^{\circ} \mathrm{C}$. It is notable that, considering the reversibility for the activation and deactivation of TRPV1 channel, ${ }^{[34]}$ the representative time point for TRPV1 activation analysis is chosen at the end of the excitation duration. Figure 5 B-C illustrates the $\Delta T$ (TRPV1 normalized activation) profile for case $15-18$, with the same NP area density at $9 \mu \mathrm{m}^{-2}$ and different excitation durations and heating powers $(g)$ at $10 \mathrm{~ns}$ and $1.69 \mu \mathrm{W}, 10 \mu \mathrm{s}$ and $0.675 \mu \mathrm{W}, 10 \mathrm{~ms}$ and $0.25 \mu \mathrm{W}, 10 \mathrm{~s}$ and $0.25 \mu \mathrm{W}$ respectively. For case $15, \mathrm{NCH}$ is observed due the short excitation duration, but there is no TRPV1 activation. As excitation duration increases, MCH is established (case 16-18), resulting in widespread TRPV1 activation (case 17\&18). Here we further quantified the TRPV1 activation by comparing the normalized activation at $\mathrm{P}_{1}$ and $\mathrm{P}_{2}$ for case 15-18. Figure $5 \mathrm{D}$ shows similar TRPV1 activation at $\mathrm{P}_{1}$ and $\mathrm{P}_{2}$ for each case, indicating either no TRPV1 activation or widespread TRPV1 activation. We further investigated a large number of conditions by altering excitation duration $(1 \mathrm{~ms}-1 \mathrm{~s})$, NP area density $\left(0.09-100 \mu \mathrm{m}^{-}\right.$

${ }^{2}$ ) and heating power $\left(10^{-2}-10^{2} \mu \mathrm{W}\right)$. Here we set a threshold of on-target TRPV1 activation (at $\left.\mathrm{P}_{1}\right)$ at $50 \%$, and off-target TRPV1 activation (at $\left.\mathrm{P}_{2}\right)$ at $10 \%$ to detect any nonspecific excitation. 
Figure $5 \mathrm{E}$ shows the threshold for on-target TRPV1 activation (blue surface) is always higher than off-target activation, indicating that on-target TRPV1 activation always comes with offtarget activation. Thus, there is always widespread TRPV1 activation by NP array heating because of the mismatch between the short excitation durations (nanosecond timescale) required for $\mathrm{NCH}$ and the millisecond timescale needed for channel activation. It should be noted, however, that it is unclear if TRPV1 can be activated at faster rates above $53{ }^{\circ} \mathrm{C}$. According to molecular dynamics simulations of the TRPV1 thermal-gating mechanism, channel opening was not observed within the $200 \mathrm{~ns}$ time frame of the simulations at temperatures of 60 and $72{ }^{\circ} \mathrm{C}$, suggesting that TRPV1 activation may be unlikely to occur at nanosecond timescale. ${ }^{[43]}$ 
A Pulsed excitation Heat generation

Protein thermal activation

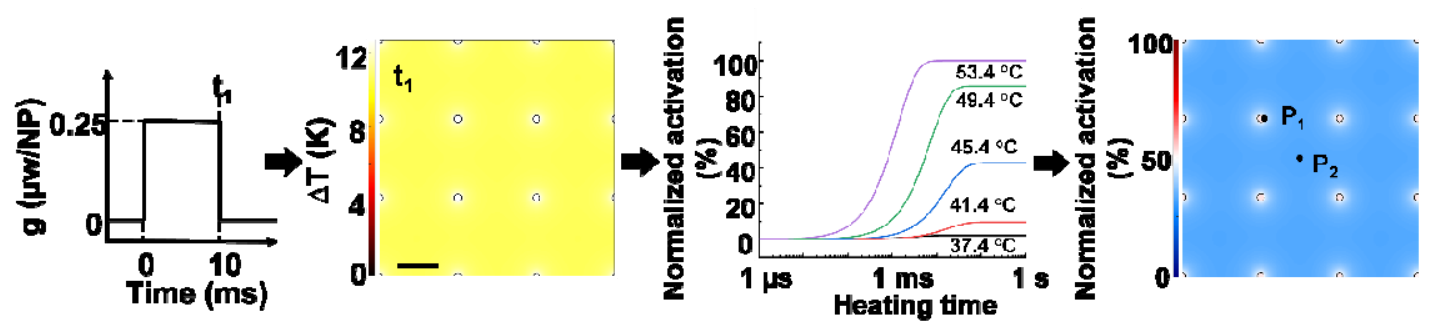

$\mathbf{B}$
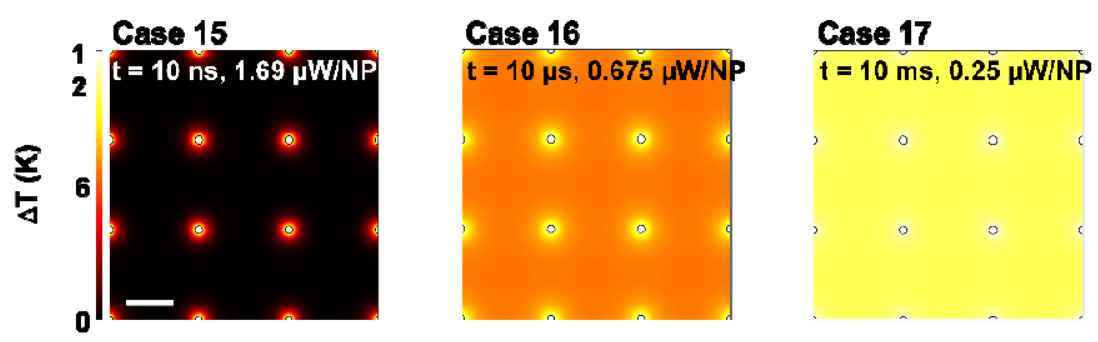

\section{Case 18}

$\mathrm{t}=10 \mathrm{~s}, 0.25 \mu \mathrm{W} / \mathrm{NP}$

C

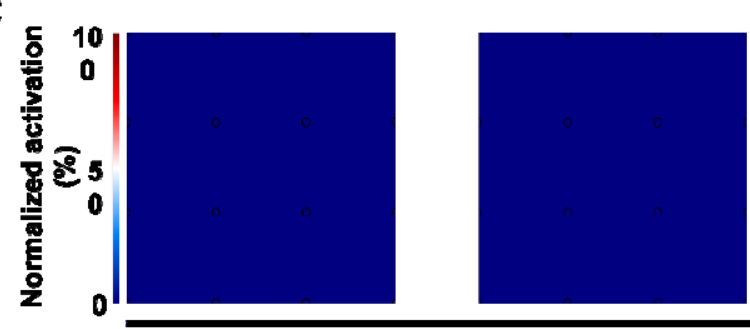

NCH but no actlvatlon

D

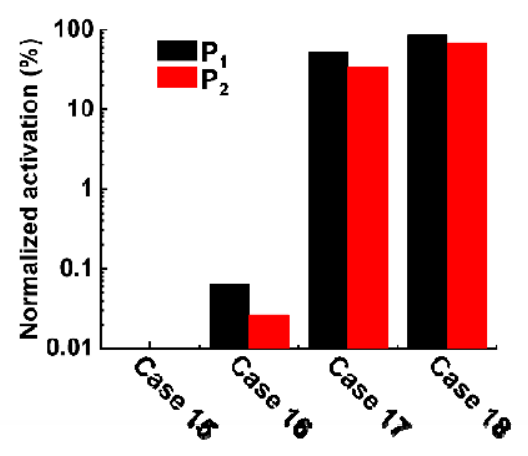

MCH with widespread actlvation

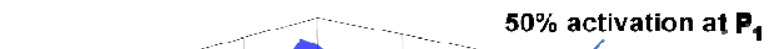

E
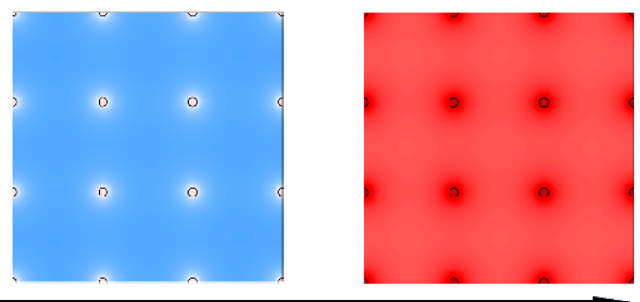

$\mathbf{5 0} \%$ activation at $\mathbf{P}_{\mathbf{1}}$

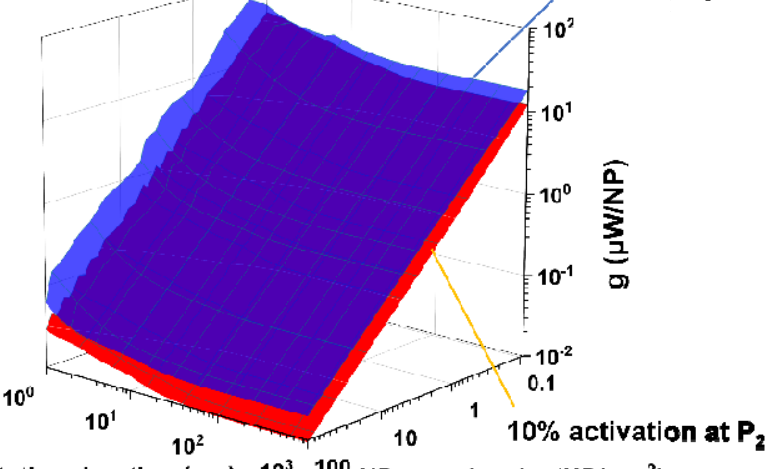

Excitation duration (ms) $10^{3} \quad{ }^{100} \mathrm{NP}$ area density $\left(\mathrm{NP} / \mu \mathrm{m}^{-2}\right)$

Figure 5. Analysis of TRPV1 channel activation by NP array heating. (A) Schematic of the analysis framework for nanoparticle (NP) array heating and the resulting TRPV1 channel activation. External excitation of the NP array heats up the NP and the surrounding media. The temperature increase drives TRPV1 channel to open, which can be described by two-state model. 
NP area density: $9 \mathrm{NP} / \mu \mathrm{m}^{2}$, excitation duration: $10 \mathrm{~ms}$, heating power: $0.675 \mu \mathrm{W} / \mathrm{NP}$. (B) $\Delta T$ profile and (C) TRPV1 normalized activation profile for cases 15-18. NP area density is 9 $\mathrm{NP} / \mu \mathrm{m}^{2}$. Excitation durations and power intensity are shown in the figure. Scalebar represents $200 \mathrm{~nm}$. (D) TRPV1 normalized activation at $\mathrm{P}_{1}$ (NP-water interface) and $\mathrm{P}_{2}$ (mid-point between particles) for case 15-18. (E) Threshold for on-target TRPV1 activation (50\%, at $\mathrm{P}_{1}$, blue surface) and off-target TRPV1 activation (10\%, at $\mathrm{P}_{2}$, red surface).

\section{Conclusion}

In this study, we investigated how single pulse heating of nanoparticle array affects biological activity, specifically protein thermal denaturation and activation. We found that excitation duration and NP area density are primary factors that determine a window for targeted denaturation. Combined with heating power, we defined quantified boundaries for targeted protein denaturation inside the heterogeneous denaturation window. On the other hand, nanoscale selective activation of the thermally-sensitive ion channel TRPV1 is not feasible based on our current understanding of its millisecond activation kinetics, and nanoparticle array heating leads to widespread TRPV1 activation. This work clearly elucidates physical limits of biological responses driven by NP heating and provides valuable guidance for designing innovative biomedical applications.

\section{ABBREVIATIONS}

NP, nanoparticle; TRPV1, transient receptor potential cation channel subfamily V member 1; $\mathrm{NCH}$, nanoscale-selective heating; $\mathrm{MCH}$, macroscale-collective heating; SLM, spatial light modulation; ROS, reactive oxygen species. 
bioRxiv preprint doi: https://doi.org/10.1101/2021.10.21.465356; this version posted October 23, 2021. The copyright holder for this preprint (which was not certified by peer review) is the author/funder. All rights reserved. No reuse allowed without permission. 


\section{REFERENCES:}

[1] J. Park, J. Huang, W. Wang, C. J. Murphy, D. G. Cahill, J. Phys. Chem. C 2012, 116, 26335.

[2] Z. Qin, J.C. Bischof, Chem. Soc. Rev. 2012, 41, 1191.

[3] A. E. Deatsch, B. A.Evans, J. Magn. Magn. Mater. 2014, 354, 163.

[4] C. M. Hu, S. Aryal, L. Zhang, Ther. Deliv. 2010, 1, 323.

[5] R. S. Riley, E. S. Day, Wiley Interdiscip. Rev. Nanomed. Nanobiotechnol. 2017, 9, 1449.

[6] E. B. Dickerson, E. C. Dreaden, X. Huang, I. H. El-Sayed, H. Chu, S. Pushpanketh, J. F. McDonald, M. A. El-Sayed, Cancer Lett. 2008, 269, 57.

[7] S. Wang, P. Huang, L. Nie, R. Xing, D. Liu, Z. Wang, J. Lin, S. Chen, G. Niu, G. Lu, Adv. Mater. 2013, 25, 3055.

[8] H. S. Huang, J. F. Hainfeld, Int. J. Nanomedicine 2013, 8, 2521.

[9] R. Singh, J. W. Lillard Jr, Exp. Mol. Pathol. 2009, 86, 215.

[10] A. M. Goodman, N. J. Hogan, S. Gottheim, C. Li, S. E. Clare, N. J. Halas, ACS nano 2017, $11,171$.

[11] D. Boyer, P. Tamarat, A. Maali, B. Lounis, M. Orrit, Science 2002, 297, 1160.

[12.] K. Jakobsohn, M. Motiei, M. Sinvani, R. Popovtzer, Int. J. Nanomedicine 2012, 7, 4707.

[13] J. C. Fraire, M. L. Masseroni, I. Jausoro, E. M. Perassi, A. M. Diaz Anel, E. A. Coronado, ACS nano 2014, 8, 8942.

[14] Y. I. Park, J. H. Kim, K. T. Lee, K. S. Jeon, H. B. Na, J. H. Yu, H. M. Kim, N.Lee, S. H. Choi, S. I. Baik, Adv. Mater. 2009, 21, 4467.

[15] D. Nelidova, R. K. Morikawa, C. S. Cowan, Z. Raics. D. Goldblum, H. P. Scholl, T. Szikra, A. Szabo, D. Hillier, B. Roska, Science 2020, 368, 1108.

[16] P. Kang, X. Li, Y. Liu, S. I. Shiers, H. Xiong, M. Giannotta, E. Dejana, T. J. Price, J. Randrianalisoa, S. O. Nielsen, ACS nano 2019, 13, 12487.

[17] R. D. Airan, R. A. Meyer, N. P. Ellens, K. R. Rhodes, K. Farahani, M. G. Pomper, S. D. Kadam, J. J. Green, Nano lett. 2017, 17, 652.

[18] H. Kang, G. H. Lee, H. Jung, J. W.Lee, Y. Nam, ACS nano 2018, 12, 1128.

[19] S. A. Stanley, J. Sauer, R. S. Kane, J. S. Dordick, J. M. Friedman, Nat. Med. 2015, 21, 92.

[20] M. A. Wheeler, C. J. Smith, M. Ottolini, B. S. Barker, A. M. Purohit, R. M. Grippo, R. P. Gaykema, A. J. Spano, M. P. Beenhakker, S. Kucenas, Nat. Neurosci. 2016, 19, 756.

[21] F. Etoc, D. Lisse, Y. Bellaiche, J. Piehler, M. Coppey, M. Dahan, Nat. Nanotechnol. 2013. $8,193$.

[22] H. Huang, S. Delikanli, H. Zeng, D. M. Ferkey, A. Pralle, Nat. Nanotechnol. 2010, 5, 602.

[23] M. Hernández-Morales, T. Shang, J. Chen, V. Han, C. Liu, Cell Rep. 2020, 30, 3250.

[24] M. Meister, Elife 2016, 5, e17210.

[25] M. I. Brier, J. W. Mundell, X. Yu, L. Su, A. Holmann, J. Squeri, B. Zhang, S. A. Stanley, J. M. Friedman, J. S. Dordick, Sci. Rep. 2020, 10, 1.

[26] P. Kang, Z. Chen, S. O. Nielsen, K. Hoyt, S. D'Arcy, J. J. Gassensmith, Z. Qin, Small 2017, 13. 1700841.

[27] E. Bergeron, C. Boutopoulos, R. Martel, A. Torres, C. Rodriguez, J. Niskanen, J. J. Lebrun, F. M. Winnik, P. Sapieha, M. Meunier, Nanoscale 2015, 7, 17836. 
[28] A. M. Wilson, J. Mazzaferri, E. Bergeron, S. Patskovsky, P. Marcoux-Valiquette, S. Costantino, P. Sapieha, M. Meunier, Nano lett. 2018, 18, 6981.

[29] J. K. Carrow, K. A. Singh, M. K. Jaiswal, A. Ramirez, G. Lokhande, A. T. Yeh, T. R. Sarkar, I. Singh, A. K. Gaharwar, Proc. Natl. Acad. Sci. U.S.A. 2020, 117, 13329.

[30] P. Keblinski, D. G. Cahill, A. Bodapati, C. R. Sullivan, T. A. Taton, J. Appl. Phys. 2006, $100,054305$.

[31] G. Baffou, P. Berto, E. Bermudez Urena, R. Quidant, S. Monneret, J. Polleux, H.

Rigneault, ACS Nano 2013, 7, 6478.

[32] G. Baffou, H. Rigneault, Phys. Rev. B 2011, 84, 035415.

[33] L. Durdevic, H. M. Robert, B. Wattellier, S. Monneret, G. Baffou, Sci. Rep. 2019, 9, 1.

[34] J. Yao, B. Liu, F. Qin, Biophys. J. 2010, 99, 1743.

[35] X. Huang, P. K. Jain, I. H. El-Sayed, M. A. El-Sayed, Lasers Med. Sci. 2008, 23, 217.

[36] S. A. Stanley, J. E. Gagner, S. Damanpour, M. Yoshida, J. S. Dordick, J. M. Friedman, Science 2012, 336, 604.

[37] X. He, J. C. Bischof, Crit. Rev. Biomed. Eng. 2003, 31, 355.

[38] H. Kim, H. Arakawa, T. Osada, A. Ikai, Ultramicroscopy 2003, 97, 359.

[39] N. Zeng, A. B. Murphy, Nanotechnology 2009, 20, 375702.

[40] G. Baffou, H. Rigneault, D. Marguet, L. Jullien, Nat Methods 2014, 11, 899.

[41] J. R. Lepock, Int. J. Hyperthermia 2003, 19, 252.

[42] K. Castillo, I. Diaz-Franulic, J. Canan, F. Gonzalez-Nilo, R. Latorre, Phys. Biol. 2018, 15, 021001.

[43] H. Wen, W. Zheng, Biophys. J. 2018, 114, 40.

\section{ACKNOWLEDGMENT:}

The research reported in this work was partially supported by the National Institute of General Medical Sciences (NIGMS) of the National Institutes of Health (award number R35GM133653), the 2019 Collaborative Sciences Award from the American Heart Association (award number 19CSLOI34770004), and the High-Impact/High-Risk Research Award from the Cancer Prevention and Research Institute of Texas (award number RP180846). The content is the sole responsibility of the authors and does not necessarily represent the official views of the funding agencies. 